\title{
Carbon Nanotube Stationary Phase in a Microfabricated Column for High-Performance Gas Chromatography
}

\author{
Takashi Nakai*, Jun Okawa, Shuji Takada, Masaki Shuzo*, Junichiro Shiomi, \\ Jean-Jacques Delaunay ${ }^{*}$, Shigeo Maruyama and Ichiro Yamada ${ }^{*}$ \\ School of Engineering, The University of Tokyo, 7-3-1 Hongo, Bunkyo-ku, Tokyo 113-8656, Japan \\ *JST, CREST, 7-3-1 Hongo, Bunkyo-ku, Tokyo 113-8656, Japan
}

\begin{abstract}
We report a microfabricated gas chromatography (GC) column that uses a thin layer of high-quality singlewalled carbon nanotubes (SWNTs) as a stationary phase. This $1.0-\mathrm{m}$-long, $160-\mu \mathrm{m}$-wide, 250 - $\mu \mathrm{m}$-deep column has the highest separation efficiency reported to date for microfabricated columns having an SWNT stationary phase. Separation efficiency was evaluated with a Golay plot, in which the minimum of the height equivalent to a theoretical plate was $0.062 \mathrm{~cm}$. The microfabricated column was able to separate n-alkanes having high boiling points under temperatureprogrammed conditions. Use of SWNTs as a stationary phase will be potentially useful for high-performance micro-GC.
\end{abstract}

Keywords: Carbon Nanotube, Stationary Phase, Gas Chromatography

PACS: 07.07.Df, 07.10.Cm, 82.80.Bg

\section{INTRODUCTION}

Conventional gas chromatography (GC) instruments using packed or capillary columns can separate most volatile compounds; however, these instruments are bulky, and their operations are power and time consuming. These drawbacks have hindered miniaturization of $\mathrm{GC}$ instruments for which a wide range of applications is foreseen [1]. Recent developments in the micro-fabrication of GC systems using micromachining technology have demonstrated the potential for reductions in size, power consumption, and analysis time compared to conventional GC systems [2-7].

A typical GC system consists of several modules, including a preconcentrator, a separation column, an oven heater, and a detector. The development and optimization of individual micromachined modules is the current trend in GC miniaturization [2]. Numerous studies have focused on miniaturizing the separation columns to develop micro-GC columns that can quickly separate samples with high efficiency [3-6]. One of the major challenges in developing micro-GC columns is the coating of the conventional polymerbased stationary phase $[7,8]$. Therefore, it is crucial to research and develop an efficient stationary phase for micro-GC columns.

Carbon nanotubes (CNTs) are attractive materials for the chromatographic stationary phase, and previous studies have demonstrated that CNTs have good separation ability due to chemical and thermal stability and a high surface-to-volume ratio [9-12]. These studies used a conventional steel, glass, or fused-silica column. A microfabricated column on a silicon on which CNTs were synthesized has also been reported $[5,6]$. These studies and reports have shown that CNTs are useful for quick temperature-programmed separation. Because CNTs grow selectively on a catalyst that can be patterned by lithography, they are better suited for integration into microfabricated columns compared to conventional polymers, which must be coated using conventional methods. Thus, CNTs have great potential for use as a stationary phase in micro-GC applications.

However, the previously reported use of CNTs as a GC stationary phase resulted in relatively low separation efficiency [5,9-12]. Moreover, gas separation columns with a CNT stationary phase suffer from poor separation of high-boiling-point compounds $[5,11,12]$. These problems are attributed to the thickness of the CNT layer.

Separation efficiency is determined by the degree of broadening of a solute band as it traverses the column. One of the sources of band broadening is solute mass transfer in the stationary phase. Thus, separation efficiency increases with a decrease in the thickness of the CNT stationary phase layer. A thin layer also promotes easy elution of highly retained 
(a) Top Si etching by DRIE

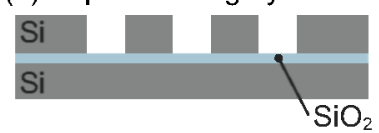

(b) Catalyst dip-coating

(c) CNT growth on $\mathrm{SiO}_{2}$

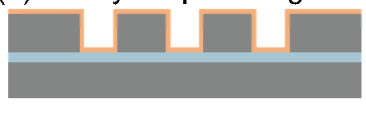

(d) Anodic bonding
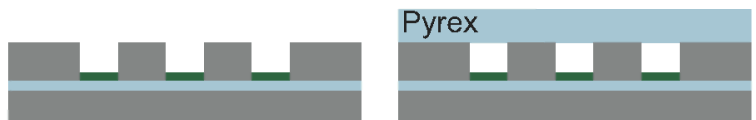

FIGURE 1. Fabrication sequence for micro-GC column with SWNT stationary phase.
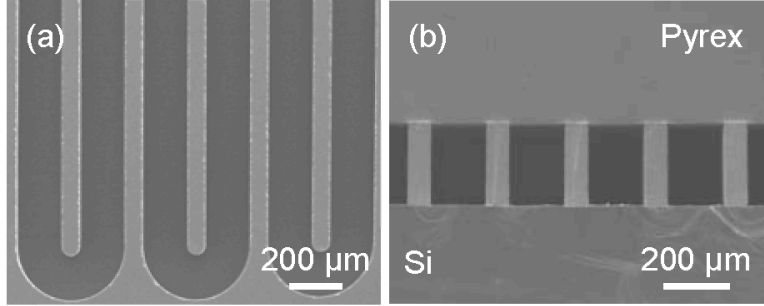

FIGURE 2. SEM images of (a) the microfabricated channel before SWNT growth and (b) the cross- section of the microfabricated column after anodic bonding.

solutes and high-boiling point compounds. Therefore, it is important to synthesize a thin layer of CNTs into microfabricated columns.

Our aim was to achieve high separation efficiency of CNT stationary phase micro-GC columns and to separate compounds with high boiling points. We used a single-walled carbon nanotube (SWNT) stationary phase synthesized into a microfabricated column. Separation efficiency was analyzed using Golay plot, and temperature-programmed chromatograms of nalkanes having high boiling points are presented.

\section{EXPERIMENTAL AND METHODS Column Fabrication}

Many microfabricated rectangular columns have been designed and fabricated by other research groups. We referred to previous work $[3,4]$ and designed a standard open tubular GC column. The length, width, and depth were $1.0 \mathrm{~m}, 160 \mu \mathrm{m}$, and $250 \mu \mathrm{m}$. It has a serpentine structure while those in previous work $[3,4]$ had a spiral one. According to a recent report [13], a serpentine GC column contributes less to band broadening than a spiral one and thus have better separation efficiency.

The low separation efficiency of previously reported CNT stationary phase columns can be attributed to the use of CNT films that are too thick, thereby increasing the resistance to mass transfer in the stationary phase, which causes band broadening [14]. A thin-film stationary phase improves separation efficiency at the cost of retention factors. Therefore, this approach is useful for improving the resolution for high-boiling-point compounds. Since CNTs have a high surface-to-volume ratio, a CNT stationary phase does not suffer from a decrease in retention factors due to a reduced film thickness. In the work reported here, CNTs were synthesized only on the bottom surface of the microfabricated column.

The steps in fabricating the open tubular GC column with a serpentine structure are illustrated in Figure 1. First, we prepared a diced SOI substrate $(250 / 3 / 320 \mu \mathrm{m})$ and etched the top Si layer using deep reactive ion etching (DRIE) to fabricate a channel (Figure 1a). After the etching, the photoresist was removed from the substrate. Next, SWNTs were grown on the bottom surface of the channel by catalytic vapor deposition (CVD); the SWNTs grew selectively from the catalytic metal particles [15]. Since the catalyst loses its activity when it is directly deposited on a silicon substrate [16], we used an SOI substrate to obtain a well-defined oxide layer on the bottom surface of the channel.

We used molybdenum and cobalt as catalytic metals for the CVD process. They were dissolved (at the same weight concentration of $0.01 \mathrm{wt} \%$ ) in ethanol to form a metal acetate solution. The solution was dipcoated on the $\mathrm{SiO}_{2}$ surface at a constant pull-up speed of $4 \mathrm{~cm} / \mathrm{min}$ at room temperature. The substrate was then dried in an oven at $400{ }^{\circ} \mathrm{C}$ for 5 minutes.

The CVD process was carried out in a quartz tube as follows (Figure 1c). The substrate was heated up to $800{ }^{\circ} \mathrm{C}$ in a furnace with a $300-\mathrm{sccm}$ flow of argon gas containing $3 \%$ hydrogen $\left(\mathrm{Ar} / \mathrm{H}_{2}\right.$ gas) so that the pressure inside the tube was kept at about $40 \mathrm{kPa}$. When the reaction temperature of $800{ }^{\circ} \mathrm{C}$ was reached, the gas flow was stopped, and ethanol vapor was introduced at a pressure of $1.3 \mathrm{kPa}$ for 10 minutes.

Finally, a Pyrex cover was sealed on the SOI substrate by anodic bonding at $350{ }^{\circ} \mathrm{C}$ and $500 \mathrm{~V}$ (Figure 1d). For the tubing connection, the microfabricated column had a 250 - $\mu \mathrm{m}$-wide opening at each end, and a 30-cm-long, 150-um-internal-diameter, 220- $\mu \mathrm{m}$-outer-diameter deactivated fused silica capillary tube was inserted into each end, and the connections were sealed with an epoxy adhesive. Figure 2 shows SEM images of the microfabricated channel before CNT growth and of a cross-section after anodic bonding.

\section{Experimental Setup}

A commercial GC was used for testing the microfabricated column. The microfabricated column was used in place of the conventional one. Helium was used as the carrier gas. The samples were injected into the built-in split/splitless injection port, and the sample vapors were detected at the column outlet using a 


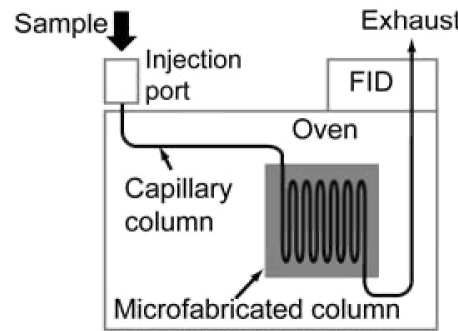

FIGURE 3. Schematic of the experimental setup used to record the chromatograms.
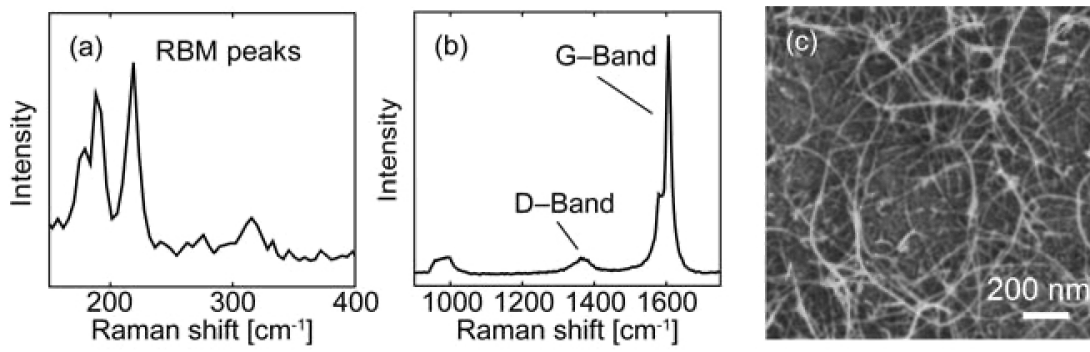

FIGURE 4. (a) Low-frequency and (b) high-frequency parts of resonance Raman spectrum for bottom surface of microfabricated channel after SWNT synthesis. (c) An SEM image of bottom surface. built-in flame ionization detector (FID), as shown in Figure 3. The temperature of the built-in oven was controlled, and the column temperature was measured with a K-type thermocouple. All chromatograms were recorded using a data logger with a sampling frequency of $50 \mathrm{~Hz}$ and an $\mathrm{A} / \mathrm{D}$ resolution of 16 bits.

The fabricated column was tested by separating $n$ alkane mixtures because CNTs are nonpolar phases [9,11]. For temperature-programmed separation, the injection volume was $0.1 \mu \mathrm{L}$ for a mixture of n-alkanes with a split ratio of $140: 1$. No solvent was used. For separation efficiency evaluation, $0.05-\mu \mathrm{L}$ samples of decane were injected with a split ratio of 140:1 using a column temperature of $40{ }^{\circ} \mathrm{C}$. The inlet and detector temperatures were set at $200{ }^{\circ} \mathrm{C}$. The average carrier gas velocities were calculated from holdup time measurements using methane injection.

\section{RESULTS Characteristics of SWNTs}

The synthesized SWNTs were characterized by resonance Raman spectroscopy and scanning electron microscopy (SEM). The SWNTs on the channel bottom of the microfabricated column were analyzed by resonance Raman spectroscopy to estimate their diameters. Figure 4 shows a typical resonance Raman spectrum. The low-frequency part of the Raman spectrum is magnified in Figure $4 \mathrm{a}$, revealing radial breathing mode (RBM) peaks, which is strong evidence of SWNT growth. The obtained RBM peaks indicate that the tube diameters were between 1 and 2 $\mathrm{nm}$ although only the SWNTs that were resonant with the excitation laser $(488 \mathrm{~nm})$ were detected. The split of the G-band peaks around $1590 \mathrm{~cm}^{-1}$ is also characteristic of SWNTs. This is highlighted in Figure $4 \mathrm{~b}$, where the high-frequency part of the spectrum is plotted. G-band peaks originate from in-plane vibrations of graphitic lattices, and D-band ones originate from defects in graphitic structures. The ratio of the G-band intensity to the D-band intensity was more than 20 , indicating the growth of high-quality SWNTs.
Figure 4c shows an SEM image of the channel bottom surface where the resonance Raman spectrum was measured. The image suggests that the thickness of the SWNT film grown inside the microfabricated column was less than those in previous work [5]. In comparison to those of previous works, the SWNT film grown using our method was thin.

\section{Column Efficiency}

We evaluated the separation efficiency of our SWNT stationary phase microfabricated column by using a plot of the height equivalent to a theoretical plate (HETP), $H$, versus the average carrier gas velocity, $u$, (Golay plot), as shown in Figure 5. The HETP values were determined for decane at a column temperature of $40{ }^{\circ} \mathrm{C}$. HETP was obtained by dividing the column length by the theoretical plate number. The theoretical plate number was calculated using the retention time for the eluted peak and its full-width at half maximum, which was estimated by Gaussian fitting. The retention factor was determined from the retention time and the holdup time, which was approximated by the travel time of methane. The retention factor was 0.6 . The minimum HETP value, $H_{\text {min }}$, found at the optimal average carrier gas velocity, $u_{\text {opt }}$, gives the maximum number of theoretical plates $N$. The trend line in Figure 5 represents the regression curve calculated using [17]:

$$
H=\frac{B}{u}+C u+D u^{2}
$$

where $B, C$, and $D$ represent the longitudinal diffusion, the resistance to mass transfer in the mobile and stationary phases, and the extra-column band broadening, respectively. At $u_{\text {opt }}, H_{\text {min }}$ calculated using Equation 1 is $0.062 \mathrm{~cm}$ and $N$ is 2500 . The smaller the HETP value, the higher the separation efficiency. Note that the $H_{\min }$ value of $0.062 \mathrm{~cm}$ is smaller than that in a previous report [5] for micro-GC column with a glass cover and an etched silicon channel with an SWNT stationary phase. To the best of our knowledge, this separation efficiency is the highest yet reported for a micro-GC column with an SWNT stationary phase. 


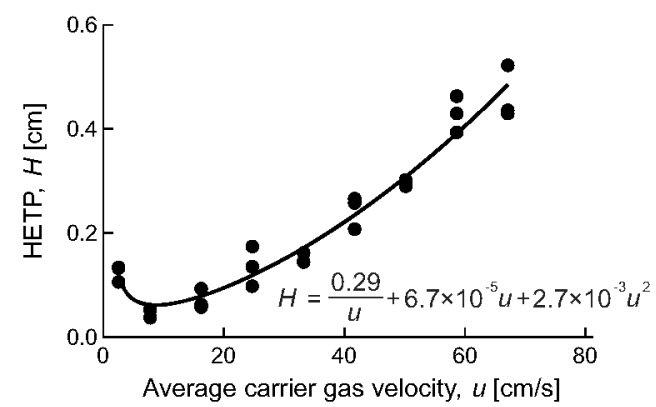

FIGURE 5. HETP versus average carrier gas velocity for micro-GC column with CNT stationary phase

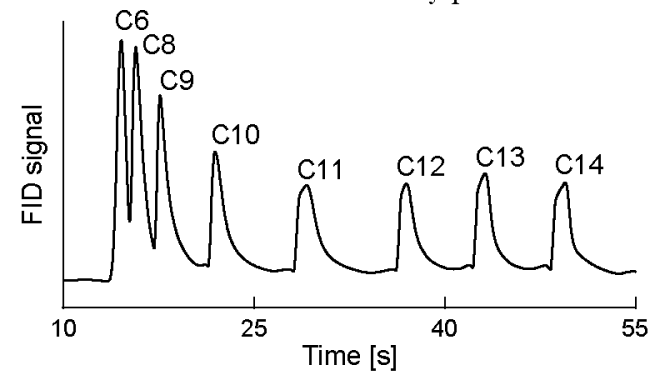

FIGURE 6. Temperature-programmed chromatogram of an n-alkane mixture. Nnumbers above peaks correspond to the alkane carbon numbers.

\section{Separation Chromatogram}

A chromatogram of the test mixture was recorded for the microfabricated column when the average carrier gas velocity was optimal under a temperatureprogrammed condition. The column temperature was increased from 30 to $105{ }^{\circ} \mathrm{C}$ at $1.4{ }^{\circ} \mathrm{C} / \mathrm{s}$. A chromatogram for the n-alkanes mixture is shown in Figure 6. All the n-alkanes were clearly separated. In previous work, the peaks for compounds having very high boiling points such as tetradecane exhibited significant tailing and band broadening [11]. Here, the peaks exhibited weaker tailing and less band broadening in spite of the much lower final column temperature $\left(105{ }^{\circ} \mathrm{C}\right)$. These results demonstrate that use of an SWNT stationary phase enables the detection of compounds having high boiling points.

\section{CONCLUSION}

We successfully synthesized a thin layer of highquality SWNTs on the channel bottom of the microfabricated column and evaluated the separation efficiency of the SWNT stationary phase microfabricated column. Using this column, we achieved the highest separation efficiency $\left(H_{\min }=\right.$ $0.062 \mathrm{~cm}$ ), reported to date, of the SWNT stationary phase microfabricated columns. We have demonstrated that the use of a thin layer of highquality SWNT stationary phase results in high separation efficiency and separation of n-alkanes with high boiling points.

\section{ACKNOWLEDGMENTS}

This work was supported by JST, CREST and GCOE, MEXT. A part of this work was conducted in Center for Nano Lithography \& Analysis, The University of Tokyo. We thank Prof. Yuji Suzuki for his valuable support in using the anodic bonder and Prof. Suguru Noda for his valuable support in using the Raman spectrometer.

\section{REFERENCES}

1. E. B. Overton et al., Field Analyt. Chem. Technol. 1,8792 (1996)

2. C. J. Lu et al., Lab Chip 5, 1123-1131 (2005).

3. M. Agah et al., J. Microelectromech. Syst. 14, 1039-1050 (2005).

4. G. Lambertus et al., Anal. Chem. 76, 2629-2637 (2004).

5. M. Stadermann et al., Anal. Chem. 78, 5639-5644 (2006).

6. V. R. Reid et al., Talanta 99, 1420-1425 (2009).

7. M. A. Zareian-Jahromi et al., J. Microelectromech. Syst. 18, 28-37 (2009).

8. G. M. Gross et al., J. Chromatogr. A 1029, 185-192 (2004).

9. Q. L. Li and D. X. Yuan, J. Chromatogr. A 1003, 203209 (2003).

10. C. Saridara and S. Mitra, Anal. Chem. 77, 7094-7097 (2005).

11. M. Karwa and S. Mitra, Anal. Chem. 78, 2064-2070 (2006).

12. L. M. Yuan et al., Anal. Chem., 78, 6384-6390 (2006).

13. A. D. Radadia et al., Proc. Transducers '07, 2011-2014 (2007).

14. V. R. Reid and R. E. Synovec, Talanta 76, 703-717 (2008).

15. S. Maruyama et al., Chem. Phys. Lett. 360, 229-234 (2002).

16. Y. Murakami et al., Chem. Phys. Lett. 377, 49-54 (2003).

17. G. Gaspa et al., Anal. Chem. 50, 1512-1518 (1978). 